\title{
28 Research Square \\ Prevalence and risk factors for death in sepsis- associated brain dysfunction: a retrospective study from ICU admissions
}

Dao-Ming Tong ( $\nabla$ tongdaoming@163.com )

Department of Neurology,Shu Yang peopole hospital,Xuzhou medical university https://orcid.org/0000-0002-4567-2439

Ye-Ting Zhou

Dp.Surgery

Shao-Dan Wang

ICU

\section{Research}

Keywords: sepsis, sepsis-associated brain dysfunction, incidence, mortality.

Posted Date: March 12th, 2020

DOI: https://doi.org/10.21203/rs.3.rs-16897/v1

License: (a) (1) This work is licensed under a Creative Commons Attribution 4.0 International License. Read Full License 


\section{Abstract}

Background: The prevalence of sepsis-associated brain dysfunction (SABD) in ICU patients with critically ill remains unknown. We are to assess whether the prevalence of sepsis in ICU would present a high prevalent life- threatening SABD. Methods: We enrolled acute critically ill adults patients from ICU (from January 1,2015 , to January 1,2017$)$. All patients were selected from onset to ICU $\leq 3$ hours and followed up to 30 day for sepsis patients who were treated in initial 48 hours or more in ICU. The predictors and risk of death of SABD was analyzed by multivariate models. Results: Of the 1349 ICU patients with acute critically ill, 748 were enrolled. Among these, the prevalence of sepsis was $48.4 \%$ (362/748). The prevalence of SABD accounted for $97.2 \%$ of sepsis (352/362), with fatality at initial 30 days was $73.6 \%$. We found that the strong clinical predictors or markers for SABD were a SIRS $\geq 2$ (OR, 3.2; 95\% Cl, 1.76.1), SOFA score $\geq 6$ (OR, 3.0; $95 \% \mathrm{Cl}, 2.6-3.5)$, and qSOFA score $\geq 2$ (OR, 0.34; $95 \% \mathrm{Cl}, 0.16-0.58)$. Cox logistic adjusted analysis revealed that lower mean arterial pressure (MAP) (OR, 1.5; 95\% Cl, 1.0-1.7) $\square$ higher SOFA score (OR, 1.8; $95 \% \mathrm{Cl}, 1.1-1.9)$, and unused a rapid antibiotics treatment in initial 3 hours $(\mathrm{OR}, 0.7 ; 95 \% \mathrm{Cl}, 0.5-0.9)$ were the predictors of the risk of death among ICU patients with SABD. Conclusions: $S A B D$ is a leading life-threatening organ dysfunction following critically ill in ICU, with an high fatality. The predictors for worse survival SABD were related to the lower MAP, higher SOFA scores, and unused a rapid antibiotic treatment within initial 3 hours.

\section{Background}

\section{Key messages}

- This study is a first to report that $97.2 \%$ of sepsis patients in ICU had a SABD and with bad outcome.

- For saving lives and reducing the prevalence of SABD, early identification of suspected bacterial infection event and for its an early rapid antibiotic treatment within initial 0.5-3 hours in ICU rather than waited SABD diagnosed is very important.

Over the past decades, sepsis as an most prevalent major disease has been become the leading cause of morbidity and mortality in ICUs worldwide[1-3]. The current estimates of 30 million new cases of sepsis and more than 6 million deaths per year around the worldwide come from a systematic review[4]. A new sepsis-3 is defined sepsis as a life-threatening organ dysfunction due to a dysregulated host response to infection[1-3]. In fact, previous studies have shown that brain dysfunction caused by sepsis included the following different terminologies: "sepsis-associated delirium (SAD)" [6], "sepsis- associated encephalopathy (SAE)" [7ه8], and "Septic encephalopathy" [9]. Actually, according to the new definition of sepsis-3, all abovemetioned term can generally be called sepsis-associated brain dysfunction (SABD), which range from initial delirium to final coma. The SAE as a mainstream of SABD has been reported frequently. The prevalence and fatality rate of SAE was over $70 \%$ of septic patients in ICU[7,8,10]. Whereas, some epidemic studies of SAE showed that a low prevalence varies from $17.7 \%$ to $53 \%$ of patients with sepsis in ICU, which is unfortunately ruled out stroke or traumatic patients[11,12]. Indeed, acute stroke and traumatic brain injury is more likely to have a secondary sepsis[2,13-15]. However, 
whether the prevalence of sepsis in ICU patients with critically ill, including acute stroke and traumatic brain injury, would present a leading high prevalence of SABD, which is still unknown. Our hypothesis was that SABD as a commonest organ dysfunction of sepsis would be presented with a high prevalence and high death risk among critically ill adults in ICU. The aim of this study was to investigate the prevalence and risk factors for death of SABD among critically ill adults in a general ICU.

\section{Methods}

\section{Study design and settings}

This is a retrospective observational study to investigate the epidemiological characteristics of critically ill adult patients with sepsis/SABD during 2 years period (January,2015-January,2017) in a general ICU in affiliated Shuyang Hospital of Xuzhou Medical University in China. The study was approved by the local ethical committee on clinical research of the hospital, and written informed consent was obtained from the nearest relative or a person who had been designated to give consent on admission of the patient.

In this study population, the subjects included acute critically ill adult patients within 3 hours from onset to the ICU, Patients who were more than 3 hours from onset to the ICU were excluded. We excluded the critically ill patients in whom did not have data in their medical records due to either death or transport out of the ICU (giving up treatment) within initial 3-6 hours. We also excluded those patients with a stay due to post-surgery/intervention.

All patients with sepsis were identified by the definition of sepsis-3 (infection plus one or more lifethreatening organ dysfunction). In this case, the SABD was identified by following 3 criteria: (1) a SABD has to present a score on the GCS of $<15$; (2) a SABD has to present a SIRS criteria $\geq 2$ and one or more organ dysfunction with evidence of infection; and (3) without evidence of direct meningitis/encephalitis and effects of sedatives. But, patients with a score on the GCS of $<15$ and organ failure but no evidence of infection were not considered sepsis. Based on the criteria for SABD in critically ill event, the study data was divided into critically ill event with SABD group and without SABD group for analysis.

\section{Definitions}

Based on the consensus of sepsis in 2016, sepsis is presented with one/more life-threatening organ dysfunction due to a dysregulated host response to infection. Thus, the SABD is defined as an acute brain dysfunction caused by sepsis, which usually follow a critically ill in the ICU and without evidence of direct meningitis/ encephalitis. This acute brain dysfunction due to sepsis can range from delirium or confusion, and seizure or focal neurological sign, as well as diffuse or multifocal neurological deficits, to final life-threatening stupor or coma.

Infection is defined as a phenomenon of systemic inflammatory reaction (or focal tissue inflammation) caused by the micro- organisms invading, settle down, or migrate to one or more elsewhere in the host. 
According to the standard definitions of the Centers for Disease Control and Prevention [16], infection is classified into community-acquired and nosocominal- acquired infection.

\section{Clinical assessments}

All demographic characteristics in patients were extracted from the ICU registration records. All clinical and laboratory data were from the hospital medical database. Most of the patients who were performed a brain CT scans were also underwent a chest CT scans on admission.

The confirmed infection is based on Angus' criteria( $(\underline{3})$. The site of infection can be defined by positive body fluid culture (including blood, peritoneal or pleural fluid, sputum fluid, urine fluid, and cerebrospinal fluid).

Because SIRS is a dysregulated host response to infection, we used the SIRS criteria $\geq 2$ for screening early suspected infection event. Moreover, the SIRS $\geq 2$ have been used to screen infection events by previous studies[5,17,18]. The SIRS criteria as following: (1) temperature $>38^{\circ} \mathrm{C}$ or $\left\langle 36^{\circ} \mathrm{C}\right.$; (2) heart rate $>$ 90 beats per minute;(3) tachypnea $>20$ respirations per minute or Pco $2<32 \mathrm{mmHg}$; (4) white blood cell count $>12.0 \times 10^{9} / \mathrm{L}$ or $<4.0 \times 10^{9} / \mathrm{L}$, or $>10 \%$ band forms. However, in this study, an early suspected bacterial infection event was identified by the two of following three points: (1) patients presented with a SIRS criteria $\geq 2$, especially WBC over $12.0 \times 10^{9} / \mathrm{L}$ or $>80 \%$ neutrophils, or $>10 \%$ band forms; (2) the infection site was identified by early thorax/abdominal cavity CT scans or initial medical records; (3) no evidence of sudden viral infectious disease or other non-bacterial infection events.

The SOFA scores were measured after critically ill event, which was assessed within 24 hours to 72 hours or more later for the ICU admissions. The acute organ dysfunction was defined as a SOFA score $\geq 2$ for a particular organ (on a scale from 0 to 4 , with $>4$ scores indicating multi-organ failure) [19]. But, we used the SOFA criteria (including the GCS score or GCS motor [GCSm] score when the patient intubated) for the brain to assess acute brain dysfunction: the GCS $=10-14$ scores as a mild brain dysfunction, GCS = 6-9 scores $(\mathrm{GCSm}<6)$ as a moderate-severe brain dysfunction (e.g., stupor or coma), and GCS $<6$ scores (GCSm<3) with no breathing as an almost irreversible brain dysfunction (e.g., deep coma or brain death). The SOFA criteria for organ dysfunctions is shown in eTable 1 in the Supplement.

The CT scans ( repeat at least one brain CT scans after coma) was assessed for patients with acute primary and secondary brain dysfunction. A downward displacement of the brain through the tentorial opening was noted in both types of herniation. Central herniation refers to a compression of the central diencephalon, radiological midline shift and peri-mesencephalic cistern compression, with clinical lethargy or coma and abnormal branstem reflexes. Uncal herniation refers to an unilateraluncal gyrus downward displacement and midbrain compression, radiological midbrain shift with third nerve compression, as well as clinical coma, pupil asymmetry $>2 \mathrm{~mm}$, and loss of reactivity to light.

\section{Outcomes analysis}


The bad outcome measure was mortality at the first 30 days. Death from critically ill event with sepsis included cardiac respiratory arrest, severe septic shock, severe respiratory failure, multiple organ dysfunction syndrome (MODS), and unexpected death. Two authors (TDM and WSD) were to assess the outcome events. To investigate the outcomes of patients at the 30 days on hospital stay, survival results were determined from the hospital records. If the length of hospitalization was less than 30 days and patient died after discharge, followed-up information was obtained from the patient's closest living relative.

\section{Statistical methods}

The results in each group were expressed as mean \pm standard deviation (SD) or medians (IQR), and $n$ (\%) for qualitative values. Fisher's exact test and the Mann-Whitney Utest were used to examine the relationship between baseline patient variables. Continuous variables were compared using Student's $t$ test. Multivariate-adjusted risk ratios (OR) and 95\% confidence intervals (Cls) were estimated using a logistic-regression model. If variables were significant in the multivariate analysis, the diagnostic values were confirmed by the receiver operating characteristic (ROC) curves analysis. Cox proportional hazards model were used to examine sepsis baseline status and to determine whether the variables played a role in the risk of death events. Survival analysis was performed using the Kaplan-Meier method. Differences between patients was considered significant if the $p$-value was $<0.05$. Statistical calculations were performed using a proprietary, computerized statistics package (SPSS 17.0.).

\section{Results}

A total of 1349 acute critically ill patients (over 18 years old) were admitted during this period. We excluded the acute critically ill patients from onset to ICU over 3 hours $(N=49)$, the patients without medical data due to death $(\mathrm{N}=248)$ and transport out of the ICU due to abandoning treatment $(\mathrm{N}=87)$ within initial 3-6 hours, the patients with a stay in ICU because of post-surgery/intervention $(\mathrm{N}=214)$, and with direct meningitis/ encephalitis $(\mathrm{N}=3)$. Finally, 748 acute critically ill patients were included in our study. Of them, $61.0 \%(456 / 748)$ critically ill patients were screened to an early suspected infection events. The prevalence of sepsis was $48.4 \%(362 / 748)$.

Baseline characteristics of the observational study for acute critically ill in ICU patients are described in Table 2 in the Supplement. Most of the patients (473/748) were males, with mean age of 59.9 years (range, 18-91 years). The median time from onset to suspected infection was in 1 hour (range, 0.5-24) and the median time from suspected infection event to sepsis/SABD event was in 9.0 hours (range, 1168). The initial median GCS scores was in 7 scores (range, 3-15).

The SABD accounted for $97.2 \%$ (352/362) of sepsis. The most common extracranial organ dysfunction of sepsis was acute respiratory failure $(81.5 \%, 287 / 362)$, followed by septic shock $(40.9 \%, 148 / 362)$, acute liver failure $(30.4 \%, 110 / 362)$, acute renal failure $(25.1 \%, 91 / 362)$. The MODS accounted for $94.8 \%$ (343/362). Epidemiological trend of SABD was similar to sepsis, with a peak in the initial first days on ICU and following weeks decreased over time (Figure 1). 
The univariate analysis of clinical characteristics in acute critically ill patients with and without SABD are described in Table 1. We found that the GCS score did not differ between the groups at initial. Moreover. We also found that there was significantly difference in age, MAP, body temperature, heart rate, respiratory rate, leukocyte count, qSOFA score, SIRS $\geq 2$ criteria, lactic acid, SOFA score, central herniation, no using antibiotics within initial $3 \mathrm{hs}$, mechanical ventilation, and LOS in ICU between the two groups (all $p<0.05)$. During 30 days follow-up, the mortality rate was also higher $(73.6 \%$ vs. $45.9 \%, p=0.005)$ among acute critically ill patients with SABD than those without SABD.

However, by multivariate logistic regression analysis, only SIRS $\geq 2$ (OR, 3.16; 95\% $\mathrm{Cl}, 1.66-6.08$ ), SOFA score $\geq 6$ (OR, 3.03; 95\% Cl, 2.57-3.54), and qSOFA score $\geq 2$ (OR, 0.34; 95\% Cl, 0.16-0.58) were established as strong predicting factors for acute critically ill patients with SABD (Table 2). The ROC curves analysis for SIRS $\geq 2$, higher SOFA score, and higher qSOFA score in patients with SABD showed that the area under the ROC curve (AUC) was $0,560(p<0.005), 0.602(p<0.001)$, and $0.544(p<0.05)$ respectively (Table 3 in the Supplement).

When the significant factors were selected into a Cox regression model, this model has shown that independent factors that affected mortality in acute critically ill patient with SABD were only lower MAP

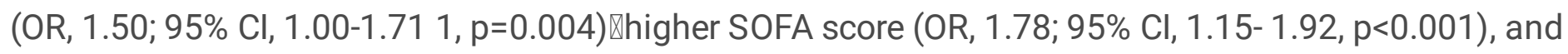
without using antibiotics treatment in initial 3 hours (OR, 0.68; 95\% $\mathrm{Cl}, 0.49-0.95, \mathrm{p}=0.022)$ (Table 3$)$.,

Kaplan-Meier curves indicate that acute critically ill patients with SABD at the first 30 days had a bad outcomes when compared to those without SABD. The Log rank for bad survival was significantly associated with acute critically ill patients with SABD at the first 30 days (Log Rank, 4.5; $p=0.033$ ). (Figure 2)

Kaplan-Meier curves indicate that acute critically ill patients who did not undergo antibiotic treatment within the first 3 hours had a bad outcome compared with those undergo antibiotic treatment within the first 3 hours. The Log rank for bad survival was significantly associated with acute critically ill patients who did not undergo antibiotic treatment within the first 3 hours (Log Rank, 25.3; $p<0.001$ ). (Figure 3 )

\section{Discussion}

This enough data observational study has mainly investigated the prevalence and risk factors for death in patients with SABD in a general ICU, although several studies have been reported on the prevalence and prognostic features for SAE in ICU[6,12,20]. Our current study shown that the prevalence of SABD was similar to the epidemiological trend of sepsis itself, suggesting that the prevalence of SABD almost represent the prevalence of sepsis.

However, critically ill patient with SABD remains a challenging diagnosis because of not be recognized "gold standard" for identify SABD. Recently, a review article for recognizing SABD indicated that SABD is a common acute brain dysfunction due to sepsis in the ICU[21]. Moreover, our study confirmed that the 
median time of SABD onset is at 9 hours after critically ill onset to suspected infection event, supporting that SABD is an acute brain dysfunction following critically ill in the ICU.

We used the multivariate logistic regression analysis to identify significant risk factors from the univariate analysis, and the results shown that only SIRS $\geq 2$, higher SOFA scores, and higher qSOFA scores were identified as the powerful clinical predictors or markers for acute critically ill patients with SABD in ICU, this was similar to previous studies[8,19,22,23]. Moreover, these findings were also demonstrated by the ROC curves analysis.

Interestingly, we found that low GCS score did not differ between the groups at initial, but our study found that SIRS $\geq 2$ \SOFA scores $\geq 6$ खand qSOFA score $\geq 2$ contributed to a SABD in patients with critically ill rather than alone low GCS score.

Importantly, Cox regression analysis indicated that the predictors for bad outcome in SABD were related to the lower MAP, higher SOFA scores, and unused a rapid antibiotics treatment within initial 3 hours in ICU. Although the effect of the lower MAP and higher SOFA scores have been recognized[12,17,20], especially higher SOFA scores for sepsis/SABD indicated MODS exist $[12,19,20]$, which was more likely to have a bad outcome or death[12,20,22,24]. However, this study shown that unused antibiotics treatment in initial 3 hours had more higher risk on death. Although there is consensus which states that rapid antibiotics treatment of sepsis is need[25], the time in rapid antibiotics treatment for sepsis is within 3 hours after SIRS onset [26]. Indeed, our patients within the first 3 hours following critically ill patients with SIRS were rarely treated with antibiotics, which result in an increasing morbidity and high risk of death in SABD. Therefore, when a suspected infection has been acquired in the community, a rapid antibiotic treatment has to be started immediately within initial $0.5-3$ hour in ICU.

The novelty of this study is a first to report that $97.2 \%$ of sepsis patients had a SABD with bad outcome, and unused a rapid antibiotic treatment within the initial 0.5 to 3.0 hours after an early suspected infection event plays a vital role in advancement of high morbidity and high risk of death in SABD. In addition, this high prevalence of SABD with bad outcome can also be explained by the following several points: (1) the low prevalence of SAE in the ICU was from a population without stroke or traumatic brain injure[11,12], whereas, our current study is included these septic patients from stroke and trauma[2,13,15]; (2) our SABD patients were generally associated with a SIRS $\geq 2$, which involves the pathogenesis of SABD contributed by Bone [27], i.e., the cytokines lead to blood brain barrier leakage and cell death [28]. A mixed SABD can be considered if accompanied by a primary brain injure; (3) most SABD in our series is presented with MODS, and the previous studies also indicated that sepsis patients with MODS were more likely to exhibit a SAE[12,21,29,30]. Therefore, early identification of this suspected bacterial infection event and for its a rapid antibiotic treatment within initial 0.5-3 hours in ICU is very important for saving lives and reducing the prevalence of SABD.

Although our data is from a prospective registration and the data analysis is available, some limitations have to be considered because of retrospective analysis. First, the studied population was from a single center so it could be biased, However, this hospital ICU is a unique regional ICU (open a 17-bed) with an 
emergency center (a total of 10 ambulances) which was responsible for all critically ill referral in this county. Thus, we believe that this sample is more likely to represent the true epidemic spectrum of critical ill, suggesting that a biased may be very small. Next, the patients with SAE were usually associated with a vasogenic brain edema or subcortical white matter ischemic lesions on imaging[31,32]. Although all of patients were performed brain CT scans, CT is more likely to be less sensitive than MRI for assessment of patients with septic brain lesions. However, MRI was less performed due to the limitation of objective reasons in the present study. Therefore, further prospective brain MR studies are needed.

\section{Conclusions}

We found that $97 \%$ of patients with sepsis in ICU had a SABD, and with high mortality at initial 30 days. The powerful clinical markers for SABD were SIRS criteria $\geq 2$, higher SOFA score, and higher qSOFA score. The predictors for bad outcome in SABD were related to the lower MAP, higher SOFA scores, and unused a rapid antibiotic treatment within initial 3 hours in ICU. The golden time of rapid completion of an antibiotic IV for saving SABD should be started immediately within the first 0.5-3 hour after the initial suspected bacterial infection event rather than waited SAE dignosed.

\section{Abbreviations}

SABD: sepsis-associated brain dysfunction

ICU: Intensive care unit

SAE: Sepsis- associated encephalopathy

SOFA: Sequential (sepsis-related) organ failure assessment

qSOFA: qick Sequential (sepsis-related) organ failure assessment

MAP: Mean arterial pressure

GCS: Glasgow Coma Scale

SIRS: Systematic inflammatory response syndrome

MODS: Multiple organ dysfunction syndrome

\section{Declarations}

\section{Availability of data and material}

The datasets used during the current study are available from the corresponding author on request.

\section{References}


1. Park DW, Chun BC, Kim JM, Sohn JW, Peck KR, Kim YS, et al. Epidemiological and Clinical Characteristics of Community- Acquired Severe Sepsis and Septic Shock: A Prospective Observational Study in 12 University Hospitals in Korea. J Korean Med Sci.2012; 27:1308- 1314.

2. Berger B, Gumbinger C, Steiner T, Sykora M. Epidemiologic features, risk factors, and outcome of sepsis in stroke patients treated on a neurologic intensive care unit. J Crit Care. 2014, 29:241-248

3. Angus DC, Linde-Zwirble WT, Lidicker J, et al. Epidemiology of severe sepsis in the United States: analysis of incidence, outcome, and associated cost of care. Crit Care Med. 2001,29 (7):1303 -1310.

4. .Fleischmann C, Scherag A, Adhikari NKJ, et al. Assessment of global incidence and mortality of hospital-treated sepsis: current estimates and limitations. Am J Respir Crit Care Med 2016; 193: 25972.

5. SingerM,DuetschmenCS,SeymourCW,etal. TheThird International Consensus Definitions for Sepsis and Septic Shock (Sepsis-3). JAMA. 2016;315:801-810.

6. Tsuruta R, Oda Y. A clinical perspective of sepsis-associated delirium. J intensive care. 2016, 4:18

7. Yonger GB, Bolton CF, Austin TW, et al. The encephalopathy associated with septic illness. Clin. Invest Med.1990,13:297-304

8. Gofton TE, Young GB.Sepsis-associated encephalopathy.Nat Rev Neurol.2012, 8:557$66.10 .1038 /$ nrneurol.2012.183

9. Tauber S.C, Eiffert H, Brück W,et al. Septic encephalopathy and septic enccephalitis. Expert Rev Anti Infect Ther. 2017 ; 15: 121- 132.

10. Sasse KC, Nauenberg E, Long A, et al. Long-term survival after intensive care unit admission with sepsis. Crit Care Med,1995, 23:1040-1047

11. Zhang LN, Wang XT, Ai YH, et al. Epidemiological features and risk factors of sepsis associated encephalopathy in intensive care unit patients:2008-2011. Chinese Medical Journal, 2012, 125: 828 $-831$.

12. Sonneville R,de Montmollin E, Poujade J, Garrouste-Orgeas M, Souweine B, Darmon M, et al. Potentially modifiable factors contributing to sepsis-associated encephalopathy. Intensive Care Med. 2017;43:1075-1084.

13. Shao IY, Elkind MSV, Boehme AK. Risk Factors for Stroke in Patients With Sepsis and Bloodstream Infections.Stroke. 2019; 50: 1046-1051. doi: 10.1161/STROKEAHA.118.023443.

14. Muehlschlegel S, Carandang R, Ouillette C, Hall W,Anderson F, Goldberg R. Frequency and impact of intensive care unit complications on moderate-severe traumatic brain injury: early results of the Outcome Prognostication in Traumatic Brain Injury (OPTIMISM) Study. Neurocrit Care.2013; 18:31831.

15. Cardozo Júnior LC, Silva RR. Sepsis in intensive care unit patients with traumatic brain injury: factors associated with higher mortality. Rev Bras Ter Intensiva;2014; 26 :148-54.

16. Horan TC, Andrus M, Dudeck MA. CDC/NHSN surveillance definition of health care-associated infection and criteria for specific types of infections in the acute care setting. Am J Infect Control. 
2008;36:309-32.

17. Donnelly JP, Safford MM, Shapiro NI, et al. Application of the Third International Consensus Definitions for Sepsis (Sepsis-3) Classification: a retrospective population-based cohort study. Lancet Infect Dis. 2017;17:661-670.

18. Bauer ME, Housey M, Bauer ST, et al. Risk Factors, Etiologies, and Screening Tools for Sepsis in Pregnant Women: A Multicenter Case-Control Study. Anesth Analg. 2019;129: 1613-1620. doi: 10.1213/ANE.0000000000003709.

19. Vincent JL, Moreno R, Takala J, et al. The SOFA (Sepsis-related Organ Failure Assessment) score to describe organ dysfunction/ failure. On behalf of the Working Group on Sepsis-Related Problems of the European Society of Intensive Care Medicine. Intensive care medicine.1996;22(7):707-10.

20. Feng Q, Ai YH, Gong H, et al. Characterization of Sepsis and Sepsis-Associated Encephalopathy. $\mathrm{J}$ Intensive Care Medicine. 2017;1:885066617719750.doi:10.1177/0885066617719750.

21. Esen F, Orhun G, Özcan PE, et al. Diagnosing acute brain dysfunction due to sepsis. Neurological Sciences .2020; 41:25-33

22. Bone RC, Balk RA, Cerra FB,et al. Definitions for sepsis and organ failure and guidelines for the use of innovative therapies in sepsis. The ACCP/SCCM Consensus Conference Committee. American College of Chest Physicians/Society of Critical Care Medicine. Chest. 1992; 101:1644-55

23. Kaukonen KM,Bailey M,Pilcher D,Cooper DJ,Bellomo R. Systemic inflammatory response syndrome criteria in defining severe sepsis. N Engl J Med.2015;372:1629-38

24. Eidelman L.A., Putterman D., Putterman C., Sprung C.L., The spectrum of septic encephalopathy definitions, etiologies, and mortalities. JAMA.1996;275:470-473.

25. Rhodes A, Evans LE, Alhazzanl W, et al. Surviving Sepsis Campaign: International Guidelines for Management of Sepsis and Septic Shock, 2016. Crit Care Med., 2017, 45:486-552.

26. Seymour CW, Gesten F, Prescott HC,et al. Time to Treatment and Mortality during Mandated Emergency Care for Sepsis. N Engl J Med.2017; 376: 2235 -2244.

27. Bone RC.Toward a theory regarding the pathogenesis of the systemic inflammatory response syndrome: what we do and do not know about cytokine regulation. Crit Care Med. 1996; 24: 163-172.

28. Shulyatnikova T, Verkhratsky A. Astroglia in sepsis associated encephalopathy. Neurochem Res.2019 Feb 18.

29. Wilson JX, Young GB. Progress in clinical neurosciences: Sepsis-associated encephalopathy: evolving concepts. Can J Neurol Sci. 2003;30:98-105.

30. Bolton CF., Young GB., Zochodne DW., The neurological complications of sepsis. Ann Neurol; 1993, 33: $94-100$.

31. Polito A, Eischwald F, Maho AL, at el. Pattern of brain injury in the acute setting of human septic shock .Crit Care. 2013;18;17: R204.

32. Sharshar T, Gray F, Poron F, Raphael JC, Gajdos P, Annane D. Multifocal necrutizing leukoencephalopathy in septic shock.Crit Care Med.2002; 30:2371-2375. 


\section{Tables}

Table 1 Univariate analysis of clinical characteristics in patients with SABD and without SABD in ICU $(n=748)$

\begin{tabular}{|c|c|c|c|}
\hline ariable & $\begin{array}{l}\text { with SABD } \\
(n=352)\end{array}$ & WithoutSABD & $(\mathrm{n}=396) \mathrm{p}$ Value \\
\hline ale gender (\%) & $211(59.9)$ & $242(61.1)$ & 0.224 \\
\hline ye (years, mean \pm SD) & $58.4 \pm 16.0$ & $61.4 \pm 15.3$ & 0.011 \\
\hline zute stroke, n (\%) & $103 \square 29.3 \square$ & $160 \square 40.4 \square$ & 0.935 \\
\hline aumatic brain injure, n (\%) & 101₫28.7๑ & $106 \square 26.8 \square$ & 0.567 \\
\hline edian time from onset to ICU (h, range) & $1.2(0.5-2.5)$ & $1.1(0.5-2.8)$ & 0.057 \\
\hline ¿S score $($ mean \pm SD) & $6.8 \pm 3.1$ & $7.2 \pm 3.0$ & 0.052 \\
\hline $\mathrm{AP}(\mathrm{mean} \pm \mathrm{SD})$ & $100.3 \pm 31.8$ & $112.0 \pm 27.4$ & 0.000 \\
\hline dy temperature $\left({ }^{\circ} \mathrm{C}\right.$, mean $\left.\pm \mathrm{SD}\right)$ & $37.8 \pm 3.8$ & $36.7 \pm 5.1$ & 0.000 \\
\hline eart rate(beats/min, mean \pm SD) & $107.2 \pm 23.3$ & $385.9 \pm 23.1$ & 0.000 \\
\hline əspiratory rate(breaths/mim,mean \pm SD) & ) $21.1 \pm 8.6$ & $18.8 \pm 5.4$ & 0.000 \\
\hline ukocyte count (x109/, mean \pm SD) & $13.8 \pm 5.7$ & $11.9 \pm 4.8$ & 0.000 \\
\hline ictic acid $(\mathrm{mmol} / \mathrm{L}$, mean $\pm \mathrm{SD})$ & $3.2 \pm 2.3$ & $2.7 \pm 2.1$ & 0.001 \\
\hline srum glucose (mmol/L,mean \pm SD) & $9.5 \pm 6.4$ & $8.9 \pm 3.5$ & 0.118 \\
\hline JFA score (mean \pm SD) & $8.4 \pm 1.9$ & $3.7 \pm 1.9$ & 0.000 \\
\hline edian qSOFA score(range) & $2.0(1-3)$ & $1.0(0-2)$ & 0.000 \\
\hline $\mathrm{RS} \geq 2, \mathrm{n}(\%)$ & $237(67.3)$ & $219(55.3)$ & 0.001 \\
\hline əntral herniation, n (\%) & $272(77.3)$ & $233(58.8)$ & 0.038 \\
\hline ncal herniation, $\mathrm{n}(\%)$ & $89(25.3)$ & $76(19.2)$ & 0.052 \\
\hline lused antibiotic within initial 3 h, n (\%) & $307(87.2)$ & $322(81.3)$ & 0.028 \\
\hline echanical ventilation,n (\%) & $287(81.3)$ & $298(75.3)$ & 0.041 \\
\hline JS in ICU (mean \pm SD) & $6.6 \pm 7.0$ & $3.0 \pm 3.4$ & 0.000 \\
\hline ortality at 30 days, $\mathrm{n}(\%)$ & $259(73.6)$ & $184(46.5)$ & 0.000 \\
\hline
\end{tabular}

Abbreviations: SABD:sepsis-associated brain dysfunction"ICU, intensive care unit; MAP, mean arterial pressure; SIRS, systemic inflammatory response syndrome; GCS, Glasgow Coma Scale; SOFA, Sequential [sepsis-related] Organ Function Assessment; LOS, length of stay;

Table 2 Logistic regression analysis to identify the early predictors factors of SABD patients in ICU $(n=748)$

\begin{tabular}{|c|c|c|c|}
\hline riable & \multicolumn{2}{|c|}{ With SAE $(n=352)$ Without SAE $(n=396)$} & OR (95\% CI) $P$ Value \\
\hline $\mathrm{RS} \geq 2, \mathrm{n}(\%)$ & $305(61.1)$ & $142(28.7)$ & $3.16(1.66-6.08)<0.001$ \\
\hline IFA scorem( mean \pm SD) & $8.4 \pm 1.9$ & $3.7 \pm$ & $3.03(2.57-3.54)<0.001$ \\
\hline OFÀ score(range) & $2.0(1-3)$ & $1.0(0-2)$ & $0.34(0.16-0.58)<0.001$ \\
\hline & & $18.8 \pm 5.4$ & $1.10(1.06-1.15)<0.001$ \\
\hline & $3.2 \pm 2.3$ & $2.7 \pm 2.1$ & $0.91(0.78-0.98) 0.017$ \\
\hline $\mathrm{S}$ in ICU (mean \pm SD) & $6.6 \pm 6.9$ & $3.0 \pm 3.4$ & $1.12(1.06-1.20)<0.001$ \\
\hline
\end{tabular}

Abbreviations: SABD:sepsis-associated brain dysfunction" SIRS, systemic inflammatory response syndrome; qSOFA, qick Sequential [sepsis-related] Organ Function Assessment; SOFA, Sequential [sepsis-related] Organ Function Assessment; LOS, length of stay; ICU, intensive care unit. 
Table 3 Cox regression analysis in acute critically ill patients with SABD and without SABD in ICU $(n=748)$.

\begin{tabular}{lrl}
\hline Variable & OR 95\% CI for ORp value \\
& $1.501 .00-1.71$ & $<0.005$ \\
Lower MAP & $1.781 .15-1.92$ & $<0.001$ \\
Higher SOFA score & Unused antibiotic within initial $3 \mathrm{~h} 0.680 .49-0.95$ & $<0.05$ \\
\hline
\end{tabular}

Abbreviations: SABD:sepsis-associated brain dysfunction" MAP, mean arterial pressure; SOFA, Sequential [sepsis-related] Organ Function Assessment; ICU, intensive care unit.

\section{Figures}

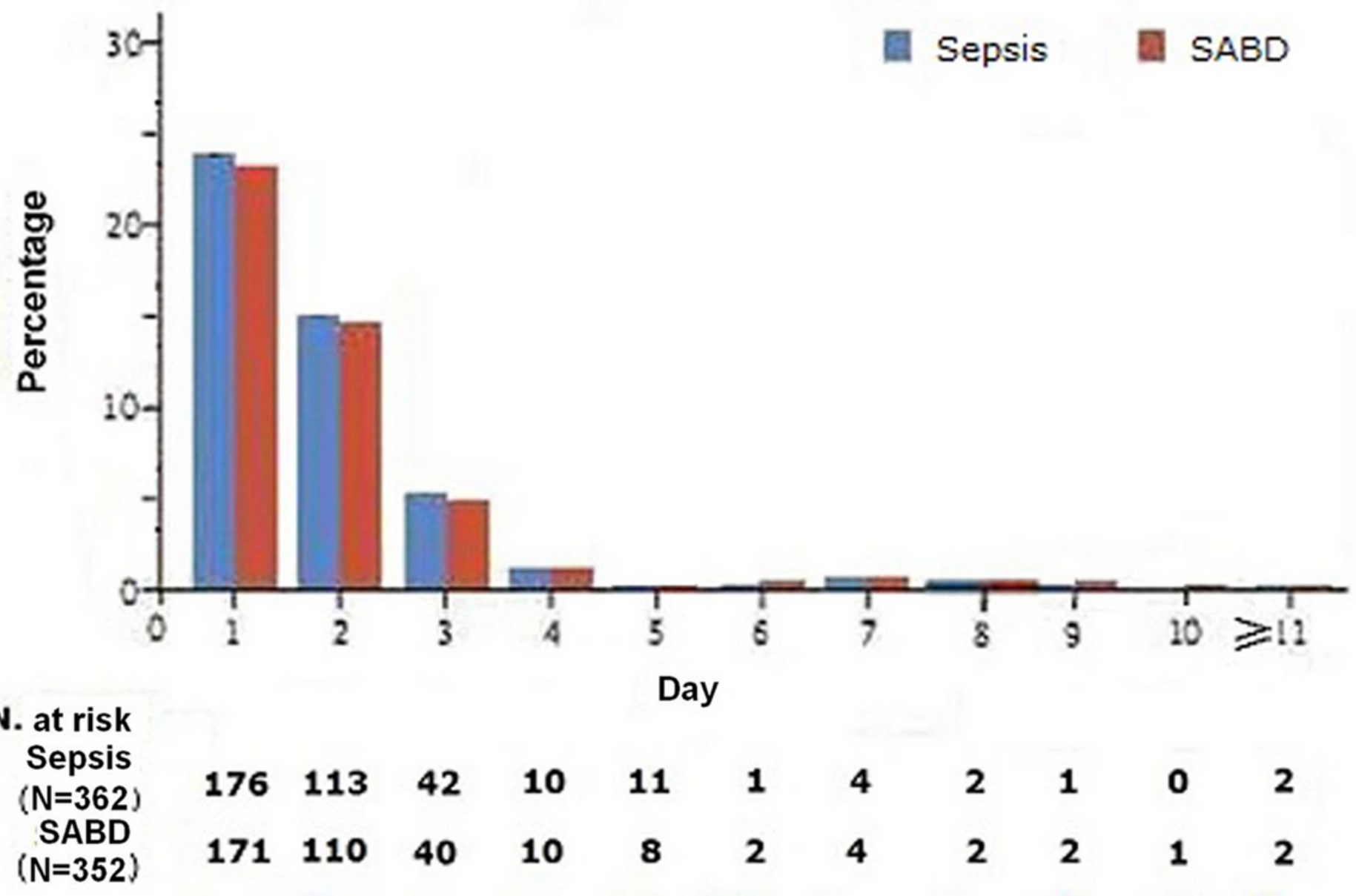

Figure 1

Epidemiological trend of SABD was similar to sepsis, with a peak in the initial first day on ICU and following weeks decreased over time. 


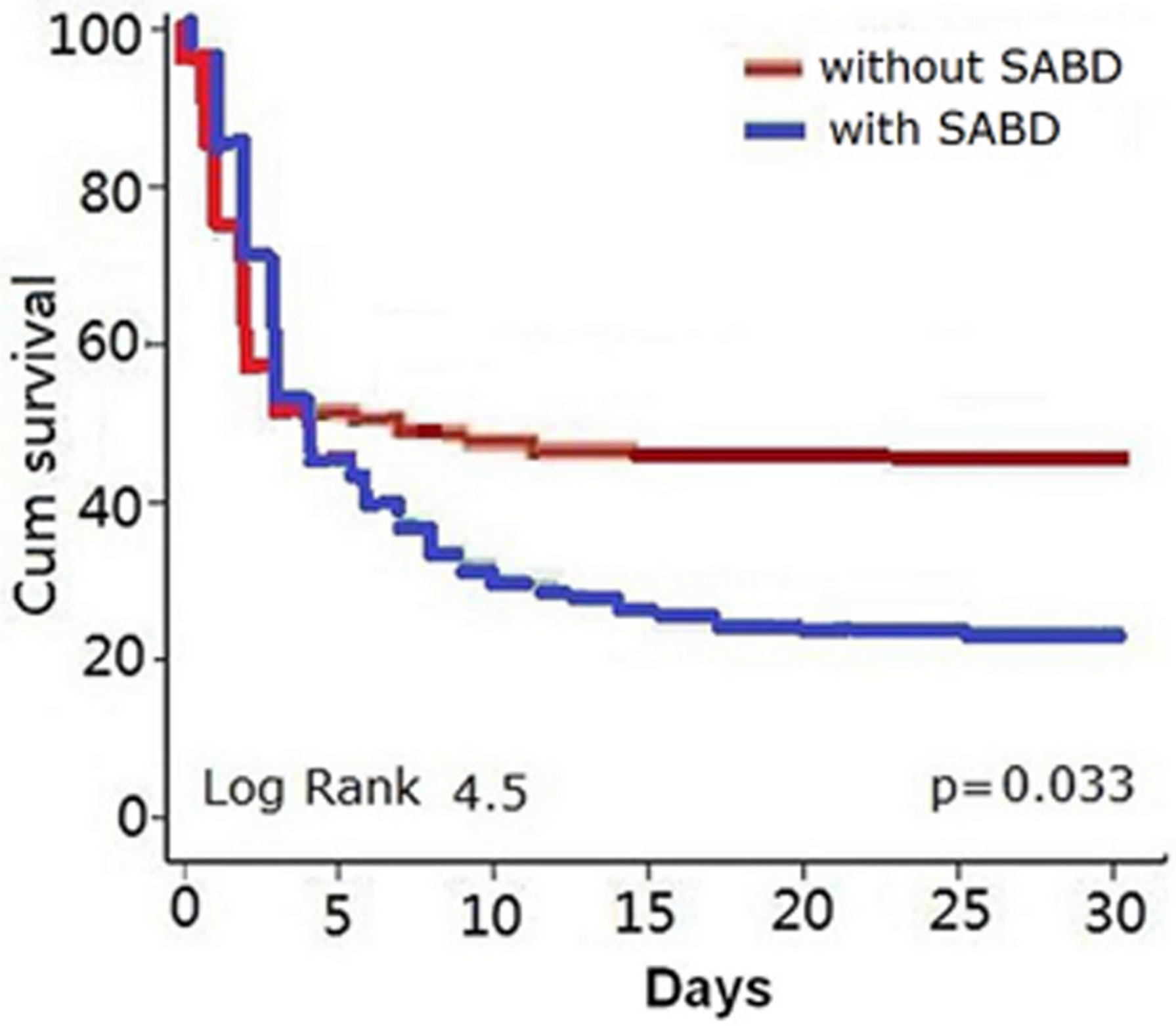

Figure 4

Kaplan-Meier survival curves that included critically ill patients with and without SABD events at the first 30 days, the worse survival was significantly associated with critically ill patients with SABD events (Log Rank, 4.5; $p=0.033)$. 


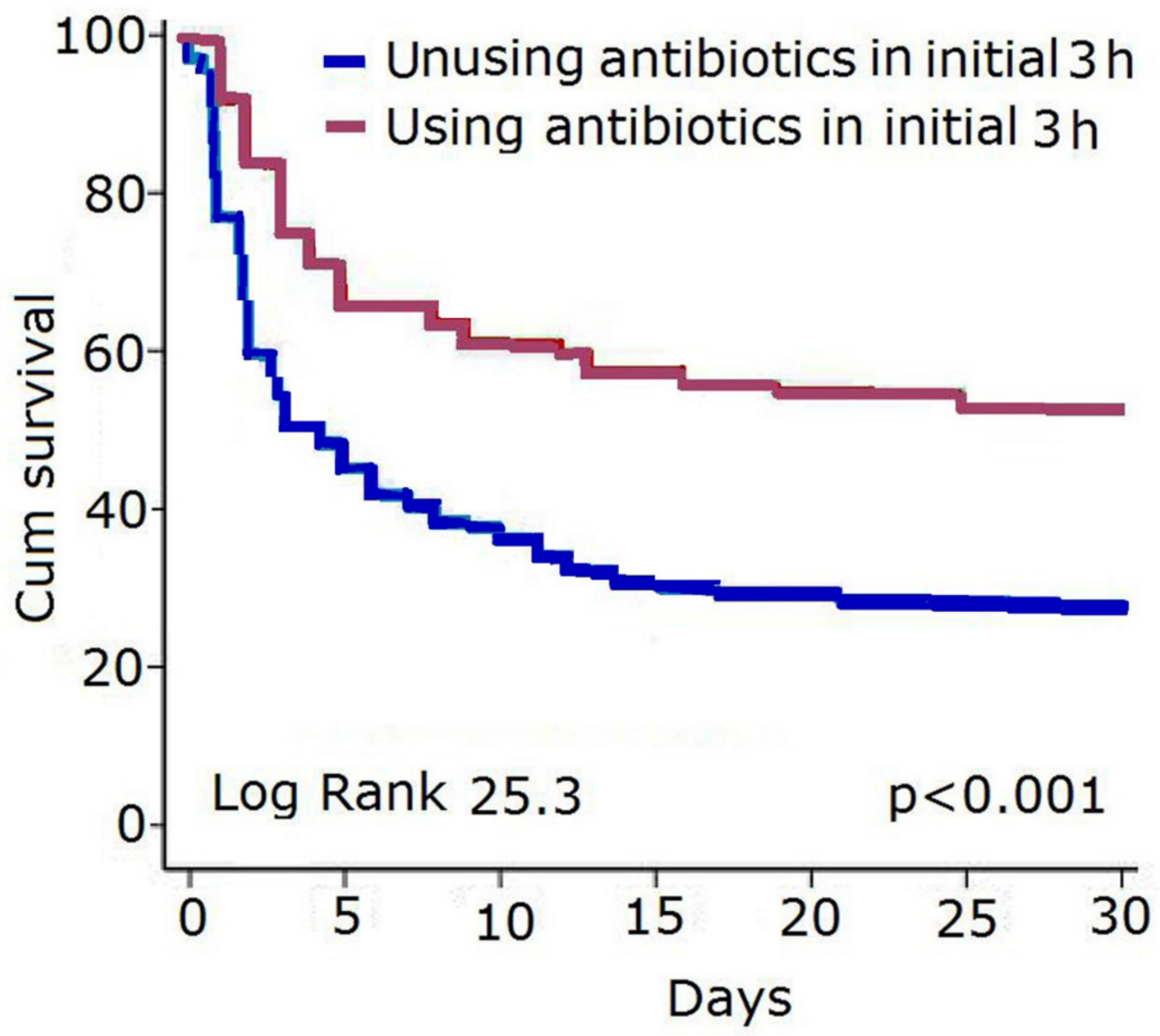

Figure 6

Kaplan-Meier survival curves showed that included critically ill patients with and without undergo antibiotic treatment within the first 3 hours following SABD. The worse survival at 30 days was significantly associated with SABD patients who did not undergo antibiotic treatment within the first 3 hours (Log Rank, 25.3; $p<0.001)$.

\section{Supplementary Files}

This is a list of supplementary files associated with this preprint. Click to download.

- SupplementaryFile.pdf 
- SupplementaryFile.pdf

Page 15/15 